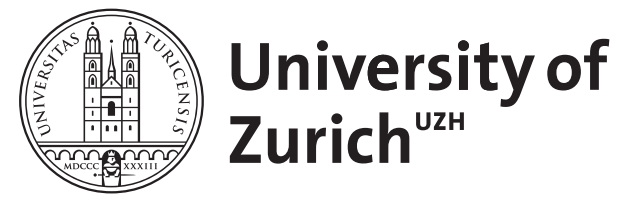

White blood cell counts in elite triathletes over four consecutive seasons

Díaz, V ; Montalvo, Z ; Banfi, G

DOI: https://doi.org/10.1007/s00421-010-1701-6

Posted at the Zurich Open Repository and Archive, University of Zurich

ZORA URL: https://doi.org/10.5167/uzh-44830

Journal Article

Published Version

Originally published at:

Díaz, V; Montalvo, Z; Banfi, G (2011). White blood cell counts in elite triathletes over four consecutive seasons. European Journal of Applied Physiology, 111(5):893-894.

DOI: https://doi.org/10.1007/s00421-010-1701-6 


\title{
White blood cell counts in elite triathletes over four consecutive seasons
}

\author{
Víctor Díaz • Zigor Montalvo • Giuseppe Banfi
}

Accepted: 13 October 2010/Published online: 3 November 2010

(C) Springer-Verlag 2010

To the editor

Exercise training leads to modification in immunological markers and white blood cell (WBC) counts. As a consequence, elite athletes can be more susceptible to infections (Gannon et al. 1997).

Horn et al. (2010) have recently reported, in a 10 years retrospective study, that triathletes and cyclists present lower WBC counts when compared with athletes of other sports. In fact, $17 \%$ of the cyclists and $16 \%$ of the triathletes showed neutropenia (values $<2.0 \times 10^{9} / \mathrm{L}$ ), and $5 \%$ of triathletes displayed also monocytopenia (values $<0.2 \times 10^{9} / \mathrm{L}$ ). These data are consistent with previous studies in endurance athletes (Lesesve et al. 2000; Parisotto et al. 2003), but longitudinal studies describing variations over several competitive seasons are still scarce in endurance sports. This is especially important in triathlon because it comprehends three different disciplines and the effects of duration/intensity of the training in one of the discipline on the other ones are poorly understood.

Communicated by William Kraemer.

V. Díaz $(\square)$

Institute of Veterinary Physiology, University of Zurich,

Winterthurerstrasse 260, 8057 Zurich, Switzerland

e-mail: diaz@vetphys.uzh.ch

\section{Z. Montalvo}

Spanish Triathlon Federation, Madrid, Spain

\section{Z. Montalvo}

Section of Internal Medicine, Endocrinology and Nutrition,

Spanish Sports Council, Madrid, Spain

G. Banfi

IRCCS Galeazzi and School of Medicine,

University of Milan, Milan, Italy
We obtained blood samples during four consecutive competitive seasons in 17 elite triathletes from the Spanish National Team (10 males, 7 females). The current age at recruitment was $23.9 \pm 4.8$ years and $23.4 \pm 4.4$ years for males and females, respectively. Following the preanalytical recommendations (Banfi and Dolci 2003), each triathlete was drawn in four different periods [start of the season, pre-competitive period, first half of the competitive period (May-July) and at the end of competitive period (September)]; and blood was analyzed using the ADVIA 120 automated system (Siemens Medical Solutions, Fernwald, Germany).

Table 1 shows WBC counts. Within gender analysis of variance (two-way ANOVA) of the log-transformed data did not show significant effect of period, season or season*period. One male and two female triathletes were characterized by leukocyte count lower than $4 \times 10^{9} / \mathrm{L}$, but no athletes showed values lower than $3.5 \times 10^{9} / \mathrm{L}$. At some period of the study, neutropenia $\left(<2 \times 10^{9} / \mathrm{L}\right)$ appeared in 5 males and 3 females, while monocytopenia $\left(<0.2 \times 10^{9} / \mathrm{L}\right)$ was displayed in 4 males and 5 females. Lymphopenia $\left(<1.0 \times 10^{9} / \mathrm{L}\right)$ appeared only in one female athlete.

Dolci et al. (2003) have previously reported an absence of variations in total leukocyte count over two consecutive seasons in professional football players. Our results confirm that WBC counts do not vary significantly in elite athletes over four competitive seasons and over the different periods (from pre-competitive to competitive period). It is important to note that our results show a chronic absence of variations of WBC counts opposite to acute variations due to high intensity training or competition (Lippi et al. 2010).

In summary, considering the results from this and previous studies (Dolci et al. 2003; Horn et al. 2010) we 
Table 1 White blood cells counts over four consecutive competitive seasons in male and female triathletes

\begin{tabular}{|c|c|c|c|c|c|c|c|c|c|c|}
\hline & \multicolumn{5}{|l|}{ Males } & \multicolumn{5}{|l|}{ Females } \\
\hline & 1 & 2 & 3 & 4 & Total & 1 & 2 & 3 & 4 & Total \\
\hline \multicolumn{11}{|c|}{ Leukocytes $\left(10^{9} / \mathrm{L}\right)$} \\
\hline Season 1 & $5.2 \pm 1.5$ & $5.8 \pm 2.5$ & $5.2 \pm 1.3$ & $5.5 \pm 1.3$ & $5.4 \pm 1.7$ & $6.6 \pm 1.5$ & $6.8 \pm 1.2$ & $6.1 \pm 0.9$ & $5.9 \pm 0.2$ & $6.4 \pm 1.1$ \\
\hline Season 2 & $5.9 \pm 1.7$ & $6.3 \pm 1.5$ & $5.3 \pm 0.6$ & $5.8 \pm 1.2$ & $5.8 \pm 1.3$ & $6.3 \pm 1.2$ & $6.9 \pm 2.8$ & $6.4 \pm 1.5$ & $7.1 \pm 2.3$ & $6.7 \pm 2.0$ \\
\hline Season 3 & $6.1 \pm 1.4$ & $5.9 \pm 1.3$ & $6.2 \pm 1.2$ & $6.5 \pm 1.8$ & $6.1 \pm 1.3$ & $5.6 \pm 1.0$ & $5.6 \pm 1.2$ & $6.1 \pm 1.5$ & $5.3 \pm 1.2$ & $5.7 \pm 1.2$ \\
\hline Season 4 & $6.7 \pm 2.1$ & $6.3 \pm 2.7$ & $5.6 \pm 1.1$ & $5.4 \pm 1.0$ & $6.1 \pm 1.9$ & $6.4 \pm 1.1$ & $6.8 \pm 0.9$ & $6.1 \pm 0.6$ & $6.2 \pm 1.6$ & $6.4 \pm 1.0$ \\
\hline
\end{tabular}

Period $F=0.33, P=0.80$; season $F=0.18, P=0.15$

Season*period $F=0.45, P=0.91$

Period $F=0.29, P=0.83$; season: $F=0.19, P=0.13$

Neutrophils $\left(10^{9} / \mathrm{L}\right)$

$\begin{array}{rllllllllll}\text { Neutrophils }(10 / \mathrm{L}) & & & & & & \\ \text { Season 1 } & 2.4 \pm 0.9 & 3.1 \pm 2.2 & 2.5 \pm 0.9 & 2.5 \pm 0.8 & 2.6 \pm 1.3 & 3.5 \pm 1.3 & 3.7 \pm 0.7 & 3.2 \pm 0.5 & 2.8 \pm 0.5 & 3.4 \pm 0.9 \\ \text { Season 2 } & 2.8 \pm 1.2 & 3.2 \pm 1.3 & 2.5 \pm 0.6 & 2.8 \pm 0.8 & 2.8 \pm 1.0 & 3.2 \pm 0.8 & 3.7 \pm 2.1 & 3.3 \pm 1.6 & 3.9 \pm 2.2 & 3.5 \pm 1.7 \\ \text { Season 3 } & 3.0 \pm 0.9 & 2.9 \pm 0.8 & 3.1 \pm 0.9 & 3.3 \pm 1.3 & 3.0 \pm 0.9 & 3.3 \pm 1.1 & 2.7 \pm 0.8 & 3.2 \pm 1.0 & 2.6 \pm 0.6 & 2.9 \pm 0.9 \\ \text { Season 4 } & 3.5 \pm 1.5 & 3.3 \pm 2.6 & 2.7 \pm 0.7 & 2.7 \pm 0.7 & 3.1 \pm 1.6 & 3.1 \pm 0.6 & 3.8 \pm 0.9 & 3.0 \pm 0.7 & 3.2 \pm 1.0 & 3.2 \pm 0.8\end{array}$

Period $F=0.35, P=0.80$; season $F=0.45, P=0.90$

Season*period $F=0.45, P=0.90$

Period $F=0.48, P=0.70$; season $F=0.73, P=0.54$

Season*period $F=0.67, P=0.74$

\begin{tabular}{ccccccccccc}
\hline Lymphocytes $\left(10^{9} / \mathrm{L}\right)$ & & & & & & & \\
Season 1 & $2.1 \pm 0.5$ & $2.0 \pm 0.4$ & $2.0 \pm 0.2$ & $2.3 \pm 0.5$ & $2.1 \pm 0.4$ & $2.4 \pm 0.6$ & $2.3 \pm 0.7$ & $2.2 \pm 0.5$ & $2.5 \pm 0.4$ & $2.3 \pm 0.5$ \\
Season 2 & $2.4 \pm 0.6$ & $2.3 \pm 0.3$ & $2.2 \pm 0.3$ & $2.3 \pm 0.4$ & $2.3 \pm 0.4$ & $2.4 \pm 0.7$ & $2.5 \pm 1.1$ & $2.5 \pm 0.8$ & $2.4 \pm 0.7$ & $2.5 \pm 0.8$ \\
Season 3 & $2.3 \pm 0.4$ & $2.2 \pm 0.5$ & $2.3 \pm 0.3$ & $2.3 \pm 0.5$ & $2.3 \pm 0.4$ & $1.7 \pm 0.7$ & $2.3 \pm 0.7$ & $2.3 \pm 1.0$ & $2.2 \pm 0.9$ & $2.1 \pm 0.8$ \\
Season 4 & $2.2 \pm 0.4$ & $2.2 \pm 0.6$ & $2.2 \pm 0.5$ & $1.9 \pm 0.2$ & $2.1 \pm 0.4$ & $2.6 \pm 0.9$ & $2.3 \pm 0.4$ & $2.4 \pm 0.6$ & $2.4 \pm 0.5$ & $2.4 \pm 0.6$
\end{tabular}

Period $F=0.19, P=0.90$; season $F=0.42, P=0.92$

Period $F=0.39, P=0.76$; season $F=1.9, P=0.14$

Season*period $F=0.44, P=0.91$

Season*period $F=0.73, P=0.68$

Monocytes $\left(10^{9} / \mathrm{L}\right)$

$\begin{array}{llllllllllll}\text { Season } 1 & 0.33 \pm 0.14 & 0.29 \pm 0.13 & 0.29 \pm 0.11 & 0.34 \pm 0.15 & 0.31 \pm 0.13 & 0.36 \pm 0.06 & 0.36 \pm 0.10 & 0.31 \pm 0.06 & 0.29 \pm 0.05 & 0.33 \pm 0.07\end{array}$

$\begin{array}{llllllllllll}\text { Season } 2 & 0.29 \pm 0.10 & 0.34 \pm 0.11 & 0.27 \pm 0.07 & 0.28 \pm 0.06 & 0.30 \pm 0.09 & 0.32 \pm 0.11 & 0.31 \pm 0.12 & 0.29 \pm 0.05 & 0.30 \pm 0.11 & 0.30 \pm 0.10\end{array}$

$\begin{array}{llllllllllll}\text { Season } 3 & 0.33 \pm 0.13 & 0.33 \pm 0.10 & 0.35 \pm 0.09 & 0.39 \pm 0.15 & 0.35 \pm 0.12 & 0.36 \pm 0.17 & 0.26 \pm 0.08 & 0.28 \pm 0.06 & 0.26 \pm 0.02 & 0.28 \pm 0.10\end{array}$

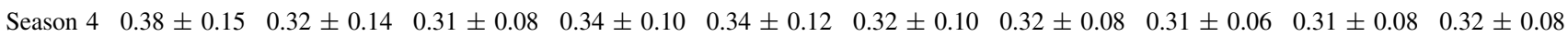

Period $F=0.28, P=0.84$; season $F=0.44, P=0.91$

Period $F=0.53, P=0.66$; season $F=2.3, P=0.86$

Season*period $F=0.44, P=0.91$

Season*period $F=0.21, P=0.99$

Values are shown as mean \pm SD. 1 Start of the season, 2 pre-competitive period, 3 first half of the competitive period, 4 end of the competitive period

suggest that fluctuations and lower neutrophil and monocyte counts in endurance athletes are more likely due to physiological adaptation rather than pathological or impaired immunological response.

\section{References}

Banfi G, Dolci A (2003) Preanalytical phase of sport biochemistry and haematology. J Sports Med Phys Fitness 43:223-230

Dolci A, Nanni G, Sisca G, et al. (2003) Leukocyte counts in professional football players. Haematologica 88: ELT31
Gannon GA, Rhind SG, Suzui M, Shek PN, Shephard RJ (1997) Circulating levels of peripheral blood leucocytes and cytokines following competitive cycling. Can J Appl Physiol 22:133-147

Horn PL, Pyne DB, Hopkins WG, Barnes CJ (2010) Lower white blood cell counts in elite athletes training for highly aerobic sports. Eur J Appl Physiol doi:10.1007/s00421-010-1573-9

Lesesve JF, Guinot M, Andolfatto S, Béné MC, Dine G (2000) Effect of elite cycling on leucocyte counts. $\mathrm{Br} \mathrm{J}$ Haematol 110:1006-1009. doi:10.1046/j.1365-2141.2000.02270-6.x

Lippi G, Banfi G, Montagnana M, Salvagno GL, Schena F, Guidi GC (2010) Acute variation of leucocytes counts following a halfmarathon run. Int $\mathrm{J}$ Lab Hematol 32:117-121. doi:10.1111/ j.1751-553X.2008.01133.X

Parisotto R, Pyne D, Martin D et al (2003) Neutropenia in elite male cyclists. Clin J Sport Med 13:303-305 